\title{
The Validity of the Column Approximation with Special Reference to the Defocusing Contrast of Small Cavities
}

\author{
BY M. RÜHLE AND M. WILKENS \\ Max-Planck-Institut für Metallforschung, Stuttgart, Germany (BRD)
}

(Received 4 June 1974)

\begin{abstract}
The diffraction contrast of lattice defects on TEM images is usually calculated by application of the column approximation (CA) which assumes basically that the wavelength of the imaging electrons approaches zero. By application of the CA the diffraction contrast can be calculated by solving ordinary differential equations (ODE) [Howie, A. \& Whelan, M. J. (1961). Proc. Roy. Soc. A 263, 217; Wilkens, M. (1964). Phys. Stat. Sol. 6, 939] rather than partial differential equations (PDE) [Wilkens (1964); Takagi, S. (1962). Acta Cryst. 15, 1311]. Since the PDE are extremely inconvenient for numerical and analytical treatments, the validity of the CA has been investigated only in a few cases [e.g., Jouffrey, B. \& Taupin, D. (1967). Phil. Mag. 16, 703]. Wilkens, A. \& Katerbau, K. -H. [Proc. 5th Europ. Reg. Conf. on Electron Microscopy, Manchester. (1972). p. 414.] have proposed a more general concept which is applicable especially to defects with localized strain fields and by which the errors of the CA are avoided to a large extent. By this method the scattering of the electrons at (and near) the defect is calculated by integrating the ODE. Behind the defect the electron wave function is developed into a superposition of Bloch waves of the perfect lattice, allowing fully for 'Fresnel' diffraction in the specimen. Furthermore, the focusing mode of the objective lens can be incorporated without additional difficulties. The method may be useful for the interpretation of the contrast of small lattice defects (diameter $\lesssim 25 \AA$ ) for which the validity of the CA becomes questionable. As an example we consider the focusing dependence of the contrast of small (strain-free) cavities [Rühle, M. \& Wilkens, M. (1972). Proc. 5th Europ. Conf. on Electron Microscopy, Manchester, p. 416], lying close to the bottom surface or close to the top surface of the transmission foil, respectively. In the first case it is confirmed that the CA is quite appropriate. In the latter case, however, significant differences were found between the CA and the "noncolumn' treatment of Wilkens \& Katerbau. First results will be published by Rühle, M. \& Wilkens, M. [Proc. 8th Intern. Congr. on Electron Microscopy, Canberra, (1974). In the press].
\end{abstract}

J. Appl. Cryst. (1975). 8, 224

\section{Studies of Lattice Defects using Weak Diffracted Beams}

\author{
By D. J. H. Cockayne, A. Gomez* and S. M. Holmes \\ Department of Metallurgy and Science of Materials, University of Oxford, England
}

(Received 20 June 1974)

\begin{abstract}
The weak-beam method of electron microscopy provides a means for studying lattice defects with greatly increased resolution compared with normal diffraction contrast techniques for a review, see Cockayne, D. J. H. [Z. Naturforsch. (1972). 27a, 452-460]\}. In particular it has enabled the dissociation of dislocations in pure f.c.c. metals and in $\mathrm{Si}$ and $\mathrm{Ge}$ to be studied in detail. In the case of $\mathrm{Si}$ and $\mathrm{Ge}$, a study has been made of the dissociation width of Shockley partial dislocations as a function of the orientation of the dislocation line. It has been found that many dislocations at and near the screw orientation show dissociation widths which are much larger than that expected from comparison with dislocations at and near edge orientation. This result will be discussed in terms of models of dislocation dissociation in the diamond-cubic structure. For studies of the size, geometry and density of pointdefect clusters, it is important to determine to what extent weak-beam images are influenced by such parameters as defect depth, foil thickness and diffraction geometry. Calculated images show that the influence of beam convergence is particularly important in this regard, because much of the dependence of the image upon defect depth and foil thickness is removed by a beam convergence comparable with that used experimentally.
\end{abstract}

\footnotetext{
* Present address: Departmento de Fisica Fundamental, Universidad de Madrid, Spain.
} 